\title{
Modeling and simulation on effects of psychological tension on the athletes' performance
}

\author{
yuehaifeng \\ (Dongying Vocational College, shandong,dongying,257091)
}

Keywords: psychological tension; athletes; athletic performance;

\begin{abstract}
Psychological factor is essential for athletes. This paper proposes a model for the effects of psychological tension on athletes' performance. In this paper, the principal component analysis method is used to quantify the psychological factors of athletes. The paper through the analysis of positive and negative factors sets impact factor to complete the analysis model. The experimental results show that it is possible to achieve better results using this model to analysis the impact on athletes' performance.
\end{abstract}

\section{Introduction}

Psychological analysis of athletes is one of the most important parts in sport psychology, which directly impact on the achievements of athletes [1]. Our athletes have serious psychological problems, which has brought great negative impact on athletic performance [2]. During the analysis, the psychological quality of athletes is the most important factor affecting performance [3]. So how to accurately analyze the impact of psychological tension on athletes' performance has become a hot issue in sport psychology research, which also aroused widespread concern of many experts and scholars[4 5].This paper proposes a model for the effects of psychological tension on athletes' performance. In this paper, the principal component analysis method is used to quantify the psychological factors of athletes. The paper through the analysis of positive and negative factors sets impact factor to complete the analysis model. The experimental results show that it is possible to achieve better results using this model to analysis the impact on athletes’ performance.

\section{Modeling for the impact of psychological tension on the performance}

The psychological tension of an athlete has great impact on his performance. Accurate analysis can effectively reduce the poor performance caused by over-anxious. The impacts described above is a non-linear, randomness and uncertainty system, whose modeling process can be expressed as follows:

(1) The psychological tension has strong non-linear property. Tension psychological factors, including the importance of the players, game state, preparing time, is a collection consisting of the above factors as described below

$$
W_{c(l+1)}=g\left(W_{c}, W_{b 1}, W_{b 2}, W_{b 3}, W_{b 4}, W_{b 5}, W_{b 6}, Q_{1}, l\right)
$$

In the formula, $l$ describes the psychological tension of an athlete, $W_{c(l+1)}$ is the negative effect on the performance, $W_{b 1} \sim W_{b 6}$ can be used to describe the impacts levels of different factors.

It can be inferred from the formula above that the factors affect athlete' psychology are complex, which can not be expressed with single linear model, so we cannot get the mapping relationship of function $f$.

(2) The psychological tension has strong randomness property. The impact equation is described as following

$$
y(l)=f([y(l), v(l), l)]
$$

The output function is 


$$
z(l)=i([y(l), v(l), l)]
$$

According to the above formulas, the changes of the athletic grades will not only closely relate to the state parameters and input data, but also relate to the data collection rates. They will also change with the states. Therefore, the impacts of the athletes' tense psychology on athletic grades are non-linear random systems. After the initial analysis on the athletes' psychology, the athletes' tense psychology data with strong correlation can be obtained. The support vector machine related theories can be used to analyze the previous data in order to predict the impacts of the athletes' tense psychology on athletic grades. Because the factors sequences of the athletic grades are one-dimension time sequences, they can be transformed to multi-dimension time sequences. The time delay factor in the athletic grades obtained impact analysis is 1 and the dimension of the time sequence is $\mathrm{n}$. The transferred multi-dimension time sequences can be described as follows.

$$
Y=\left|\begin{array}{cccc}
y_{11} & y_{12} & \cdots & y_{1 n} \\
y_{21} & y_{22} & \cdots & y_{2 n} \\
\cdots & \cdots & \cdots & \cdots \\
y_{p 1} & y_{p 2} & \cdots & y_{p n}
\end{array}\right| Z=\left|\begin{array}{c}
z_{1 n} \\
z_{2 n} \\
\cdots \\
z_{p n}
\end{array}\right|
$$

According to relevant data of psychological tension and factors affecting performance, we can estimate the impact of psychological tension on performance. Actually, the estimation calculated from the support vector machine is a relationship between the input data and output data, which is show as following formula

$$
z_{j}=g\left(y_{j}\right), g: S^{p} \rightarrow S
$$

In the above formula, $S^{p}$ is used to describe the factors affecting sports performance

The impact model obtained according to support vector machine theory is shown as follows:

$f(y)=\sum_{j=1}^{l}\left(\beta_{j}-\beta_{j}^{*}\right) \exp \left|\frac{\left\|y-y_{j}\right\|^{2}}{2 \varsigma^{2}}\right|+c$

Solving the above problems, it is possible to get the data processor, input data, output data and the corresponding kernel functions of the model.

According to the theory of discrete wavelet transform, the following formula is used to reconstruct the above model

$$
d_{k-1}=d_{k} \bar{i}+e_{k} \bar{h}(k=0,1,2, \cdots, M)
$$

In the above formula, $\bar{i}$ can be used to describe the dual operator of $i, \bar{h}$ can be used to describe the dual operator of $h$.

According to the support vector machine theory, we can effectively estimate the correlations between athlete's performance and psychological tension. Reconstructed by discrete wavelet, the psychological tension impact on athlete's performance can be obtained.

\section{Simulation results}

\subsection{Data sources}

To validate the effectiveness of the proposed algorithm, an experiment is carried out. In the experiment, we choose athletes' record data in a sports school in several years as basic data, and then use MATLAB7.0 to get results.

\subsection{Simulation results}

In the experiment, we need to rationalize the use of athletic data, choose the most important data as initial data. We respectively use the traditional modeling algorithm and the proposed one to analyze the impact of psychological tension on athlete's performance. To evaluate the performance, we uses table one to record the related results. 
Table 1 The results comparison table

\begin{tabular}{cccc}
$\begin{array}{c}\text { Test } \\
\text { sample } \\
\text { s }\end{array}$ & $\begin{array}{c}\text { Actu } \\
\text { al } \\
\text { value }\end{array}$ & $\begin{array}{c}\text { The } \\
\text { traditional } \\
\text { algorithm }\end{array}$ & $\begin{array}{c}\text { The } \\
\text { improved } \\
\text { algorithm }\end{array}$ \\
\hline 1 & 0.864 & 0.662 & 0.811 \\
2 & 0.856 & 0.532 & 0.833 \\
3 & 0.708 & 0.456 & 0.744 \\
4 & 0.433 & 0.425 & 0.449 \\
5 & 0.735 & 0.702 & 0.742 \\
6 & 0.361 & 0.295 & 0.381
\end{tabular}

It can be inferred from the table that in the 6 testing, the modeling result of the proposed algorithm is closer than that of the traditional algorithm.

\section{4 conclusions}

To avoid the defects of the traditional algorithm, this paper proposes a model for the effects of psychological tension on athletes' performance. In this paper, the principal component analysis method is used to quantify the psychological factors of athletes. The paper through the analysis of positive and negative factors sets impact factor to complete the analysis model. The experimental results show that it is possible to achieve better results using this model to analysis the impact on athletes’ performance

\section{references}

[1] Bo Ling GANG etc. Based on GSM traffic modeling and prediction of seasonal ARIMA model [J]. Journal of mathematical statistics and management, 2004, 11 (6) : 19-24.

[2] battlefield munitions-mr C.K emelmethods a survey of the current techniques [J]. Journal of Neurocomputing. 2002.48 (1-4) : 63-84.

[3] Hu Huang, li lei. Improved regression algorithm application in telecommunication traffic prediction [J]. Computer engineering and application, 2001, 3 (3) : 79-82.

[4], establish resplendence ardently $\mathrm{Xu}$ Baosong $\mathrm{Wu}$ Jinkun etc. Based on least squares support vector machine (SVM) dam mechanical parameters inversion [J]. Journal of geotechnical engineering. 2008.30 (11) : 1722-1725.

[5] hong-fa ke, ringo chan, jin-liang wu. Nonlinear grey prediction of airborne electronic equipment spare parts planning model [J]. Journal of systems engineering and electronics, 2008, 30 (3) : 503-505. 